\title{
What is a supercoiling-sensitive gene? Insights from topoisomerase I inhibition in the Gram-negative bacterium Dickeya dadantii
}

\author{
Maïwenn Pineau*,1, Shiny Martis B.*,1, Raphaël Forquet ${ }^{1}$, Jessica Baude ${ }^{1}$, Lucie Grand ${ }^{2}$, Florence \\ Popowycz ${ }^{2}$, Laurent Soulère ${ }^{2}$, Florence Hommais ${ }^{1}$, William Nasser ${ }^{1}$, Sylvie Reverchon ${ }^{1}$ and Sam \\ Meyer ${ }^{1, \mathrm{a}}$ \\ * Equal first authors \\ ${ }^{1}$ Université de Lyon, INSA Lyon, Université Claude Bernard Lyon 1, CNRS UMR5240, Laboratoire de \\ Microbiologie, Adaptation et Pathogénie, 69621 Villeurbanne, France \\ ${ }^{2}$ Université de Lyon, INSA Lyon, Université Claude Bernard Lyon 1, CPE Lyon, CNRS UMR 5246, Institut \\ de Chimie et Biochimie Moléculaires et Supramoléculaires, 69622 Villeurbanne, France \\ ${ }^{a}$ To whom correspondence should be addressed. Tel: +33 4724385 16; Email: sam.meyer@insa-lyon.fr
}

\section{Abstract}

DNA supercoiling is an essential mechanism of bacterial chromosome compaction, whose level is mainly regulated by topoisomerase I and DNA gyrase. Inhibiting either of these enzymes with antibiotics leads to global supercoiling modifications and subsequent changes in global gene expression. In previous studies, genes responding to DNA relaxation induced by gyrase inhibition were categorized as "supercoiling-sensitive". Here, we studied the opposite variation of DNA supercoiling in the phytopathogen Dickeya dadantii using the non-marketed antibiotic seconeolitsine, and obtained the first transcriptomic response of a Gram-negative bacterium to topoisomerase I inhibition. We find that the responding genes essentially differ from those observed after DNA relaxation, and further depend on the growth phase. We characterised these genes at the functional level, and also detected distinct patterns in their spatial and orientational organisation along the chromosome. Altogether, these results suggest that the "supercoiling-sensitivity" is not an intrinsic property of promoters, but depends on the action of specific topoisomerases, on the physiological conditions, and on their genomic context. Based on previous in vitro expression data of several promoters, we propose a qualitative model of SC-dependent regulation that accounts for many of the contrasting transcriptomic features observed after gyrase or topoisomerase I inhibition.

\section{Introduction}

DNA supercoiling (SC) is the product of torsional stress ubiquitously experienced by the double-helix in all kingdoms of life. In bacteria, the chromosome is maintained in a steady-state level of negative SC by the interplay of nucleoid associated proteins (NAPs) and the activity of topoisomerases. The DNA gyrase (belonging to class II topoisomerases) introduces negative supercoils by ATP-dependent double-strand cleavage; conversely, topoisomerase I (TopoI, class IA) removes excessive negative supercoils through ATP-independent single-strand cleavage, and topoisomerase IV (TopoIV, class II) through ATP-dependent double-strand cleavage (1-3). Cells react to SC imbalances by a fast adjustment of topoisomerase activities, according to a homeostasis mechanism (4). This balance plays a key role in many cellular functions, and in particular in the expression of the genome, which is our focus in this study. 
The presence of torsional stress in the DNA template is known to affect the transcription process at several successive steps: by modulating the binding of transcriptional regulators and RNA Polymerase (RNAP) itself, the formation and stability of the open complex (5), promoter clearance (6), elongation and termination $(7,8)$. As a result, SC was soon recognized to act as a global transcription factor $(9,10)$, although the complexity of the underlying mechanisms made it difficult to estimate to what extent this action was used by the cell as a bona fide global regulatory mechanism, or it was just a side-effect of other physiological functions of chromosome compaction. Early studies demonstrated a strong regulatory action of SC on the promoters of stable RNAs in Salmonella enterica and Escherichia coli $(5,11)$, pointing to a role in growth control (10) consistent with the close relationship between SC and the cell metabolism (12). But other promoters were found to be equally affected $(7,13)$, which was then confirmed and broadened by high-throughput transcriptomics methods (14-16). In analogy to the "regulons" of transcriptional factors, these promoters were often termed "supercoiling-sensitive", although that notion remains poorly defined, considering the lack of clearly identified sequence determinants (17), and the variability in the response of many promoters to SC alterations depending on their context and the experimental protocol of the assay. For example, the lacP promoter of $E$. coli is strongly repressed by DNA relaxation in vitro $(7)$, but is unaffected in vivo $(14,15)$; the proportion of genes activated by DNA relaxation in $S$. enterica varied between $70 \%$ in a random fusion assay (18) and $27 \%$ in a RNA-Seq transcriptome (19).

In vivo, these responses to $\mathrm{SC}$ variations were obtained by two distinct methods (20). The expression level can be measured in topoisomerase mutant strains, which usually exhibit a different SC level than the parental strain cultured in the same conditions $(20,21)$; however, the difference in promoters' expression then reflects not only the direct regulatory effect of SC, but also that of the resulting global change in transcriptional regulatory activity in the mutant strain, and these two contributions are difficult to distinguish. To avoid this issue, it is often preferred to use a wild-type strain, and induce a rapid SC variation by applying topoisomerase inhibiting antibiotics $(8,20)$. Commonly used drugs belong to the coumarin family (coumermycin, novobiocin), inhibiting the ATPase activity of gyrase (with a weak effect on TopoIV), and the quinolone family (norfloxacin, ciprofloxacin, oxolinic acid) inhibiting the ligase activity of gyrase and TopoIV $(1,3,22)$. These drugs induce a sudden DNA relaxation in various species, whose effect on gene expression can then be measured. The main shortcoming is that they also trigger SC-independent stress-response pathways in the cell, which are then difficult to distinguish from SC-induced transcriptional effects; this is especially true with quinolones, which trigger double-strand breaks and a SOS response with pleiotropic effects. To minimize these issues, the drug concentration is usually kept as low as possible, and the expression is measured very quickly after the shock. Still, in order to characterize specifically the effect of SC on transcriptional regulation, it is thus highly desirable to increase the robustness of the analysis by comparing the expression patterns obtained with different methods (15). In this respect, a major limitation of existing studies is that, since gyrase is the primary target of all these drugs in Gram-negative bacteria, the transcriptomic response was analysed only in one direction, DNA relaxation, introducing a strong bias in the analysis of the SC-sensitivity of promoters.

The opposite variation could also be induced by applying quinolones on engineered strains harbouring mutant gyrase genes, where only the (weaker) relaxing activity of TopoIV is inhibited by the drug $(2,23)$. However, the steady-state SC level of these strains differs from that of wild-type 
ones, and the associated transcriptomic response was never monitored. In wild-type cells, TopoI seemed a particularly suitable drug target (24), both in clinical research as it is the only enzyme of type IA topoisomerases family in many pathogenic species, but also as a way to study the effect of $\mathrm{SC}$ in transcriptional regulation, since this enzyme plays a direct role in the handling of torsional stress associated with transcription, while TopoIV is predominantly involved in replication (25). Additionally, not only was TopoI the first topoisomerase to be discovered (26), but the reduction or loss of its activity due to mutations in the topA gene in E. coli and $S$. enterica played an important role in the early discovery of the reciprocal interaction between transcription and SC (21, 27-29). In recent years, many compounds were shown to act as TopoI inhibitors with unequal effectiveness as antimicrobial agents (24). In particular, one of them named seconeolitsine (30) is effective against the Gram-positive bacterium Streptococcus pneumoniae, and at low concentrations, induces a transient SC increase associated with a global change in the transcriptional landscape (31).

Here, we show this drug to be equally effective in Gram-negative bacteria, and we use it to report the first transcriptomic response to TopoI inhibition and resulting SC increase in Gram-negative bacteria, using the phytopathogen Dickeya dadantii as a model. The latter is attractive for this analysis, not only because of its proximity to the laboratory models E. coli and S. enterica, but also because SC was shown to be a strong regulator of its key virulence genes $(16,32)$, and since SC-affecting environmental signals are known to be influential in its infection process, in particular osmolarity variations resulting in an increase of the cellular SC level $(16,32)$. Deciphering the mechanisms of SC-related transcriptional regulation in that species is thus important for our understanding of the mechanisms of virulence, as well as of transcriptional regulation as a general process.

In the following, we first demonstrate the inhibitory effect of high concentrations of seconeolitsine on $D$. dadantii growth and then show that when applied at lower concentration, a seconeolitsine shock quickly increases the cellular SC level. We analyse the effect of this shock on the expression of the genome, and in particular, we illustrate the patterns of spatial gene organisation manifested in the transcriptional response. By comparing this response with that of the gyrase inhibitor novobiocin and based on these contrasting observations, we propose a qualitative model explaining many notable features possibly involved in defining the "supercoiling-sensitivity" of promoters.

\section{Materials and Methods}

\section{Seconeolitsine synthesis}

Seconeolitsine was obtained from boldine in a two steps synthesis as previously described (33). In a first step, a reaction of demethylation was conducted in acidic conditions followed by a reaction with dibromomethane. The intermediate neolitsine was then reacted with chloroethyl chloroformate in dichloroethane followed by aromatization and ring opening achieved in refluxing methanol (Supplementary Fig. S1).

\section{Inhibitory action of seconeolitsine}

D. dadantii 3937 were grown at $30^{\circ} \mathrm{C}$ in microplates containing Luria-Broth medium and increasing concentrations of seconeolitsine dissolved in DMSO (5 or $10 \mathrm{mM}$ stock solution, keeping the final volume of DMSO below 4\%). Optical densities were recorded every 5 min using an automatic microplate reader (Tecan Spark), and growth curves were fitted to a Gompertz equation to estimate growth rates and time lags (34). 


\section{Bacterial cultures for seconeolitsine shock}

D. dadantii 3937 were grown at $30^{\circ} \mathrm{C}$ in $\mathrm{M} 63$ supplemented with sucrose at $0.2 \%$ (wt/vol) until the exponential $\left(\mathrm{OD}_{600}=0.2\right)$ or transition to stationary phase $\left(\mathrm{OD}_{600}=1.1\right)$. Cells were then shocked with seconeolistine dissolved in DMSO at $50 \mu \mathrm{M}$ during $5 \mathrm{~min}$ (RT-qPCR experiments) or $15 \mathrm{~min}$ (RT-qPCR and RNA-Seq experiments). An additional control was performed with pure DMSO for RT-qPCR experiments.

\section{Topoisomer separation in chloroquine-agarose gels}

The topoisomer distribution was analysed as previously described (35). Reporter plasmids pUC18 were transformed into D. dadantii 3937. 15 minutes after the shock, plasmids were extracted with the Qiaprep Spin Miniprep kit and migrated on a 1\% agarose gel containing $2.5 \mu \mathrm{g} . \mathrm{ml}^{-1}$ chloroquine at $2.5 \mathrm{~V} . \mathrm{cm}^{-1}$ for $16 \mathrm{~h}$. Under these conditions, more negatively supercoiled migrate faster in the gel. Chloroquine gels were subjected to densitometric analysis using Image Lab 6.0 software (Biorad). Distributions of topoisomers were normalised and quantified in each lane independently.

\section{RNA extraction}

Total RNAs were extracted either with the frozen-phenol method (36) (RNA-Seq experiments) or with the Qiagen RNeasy Plus Mini Kit, including a bacterial lyse with a lysozyme solution at 1 mg. $\mathrm{ml}^{-1}$ and the optional DNase treatment (RT-qPCR experiments). The absence of genomic DNA contamination was further verified by PCR amplification with the Lucigen EconoTaq PLUS GREEN and $r y h B$ primers (Tab. S1), following manufacturer's instructions. When necessary, an additional DNase treatment was performed using the BioLabs DNase I to ensure RNA purity.

Extracted RNAs were quantified using a ND-1000 NanoDrop spectrophotometer. RNA quality was checked by agarose gel electrophoresis.

\section{Quantitative real time PCR}

$1 \mu \mathrm{g}$ of total RNAs were reversed transcribed using the Thermo Scientific RevertAid First Strand cDNA Synthesis Kit. Reaction mixes were incubated at $25^{\circ} \mathrm{C}$ for $5 \mathrm{~min}, 42^{\circ} \mathrm{C}$ for $60 \mathrm{~min}$ and $70^{\circ} \mathrm{C}$ for $5 \mathrm{~min}$.

The quantitative PCR was carried out using the Thermo Scientific Maxima SYBR Green/ROX qPCR Master Mix with the LC480 Lightcycler from Roche and the primers listed in Tab. S1. The following thermal cycling reactions were executed: (i) an initial denaturation step at $95^{\circ} \mathrm{C}$ for 10 min, (ii) 45 amplification cycles at $95^{\circ} \mathrm{C}$ for $15 \mathrm{~s}, 58^{\circ} \mathrm{C}$ for $30 \mathrm{~s}$ and $72^{\circ} \mathrm{C}$ for $40 \mathrm{~s}$. The housekeeping gene rpoA was used as a normalizer for the gene expression ratios. The uniqueness of the amplification product is verified with the melting curve.

\section{RNA Sequencing}

All samples were collected in two biological replicates ( 8 samples in total). Steps of ribosomal RNA depletion, cDNA library preparation and high-throughput sequencing were carried by the MGX Montpellier GenomiX platform, using the Illumina TruSeq stranded mRNA sample preparation kit and HiSeq2500 sequencing providing 50-nt single-end reads. They were mapped on the reference genome of $D$. dadantii 3937 (NCBI NC_014500.1) with Bowtie2 and counted with htseq-count. Gene differential expression analysis was performed with DESeq2 with a threshold of 0.05 on the adjusted p-value. 


\section{Statistics and data analysis}

All statistical analyses and graphs were made with a homemade Python code. Error bars are 95\% confidence intervals. Proportions of activated genes among differentially expressed genes were compared with $\chi^{2}$-tests. The orientation of a gene is defined relative to the orientation of its neighbors (either convergent, divergent or tandem). Functional enrichment was analysed using the Gene Ontology classification (37). Only functions corresponding to at least four $D$. dadantii genes were considered. Chromosomal domains were previously defined in (16).

\section{Results}

\section{High concentrations of seconeolitsine impede $D$. dadantii growth}
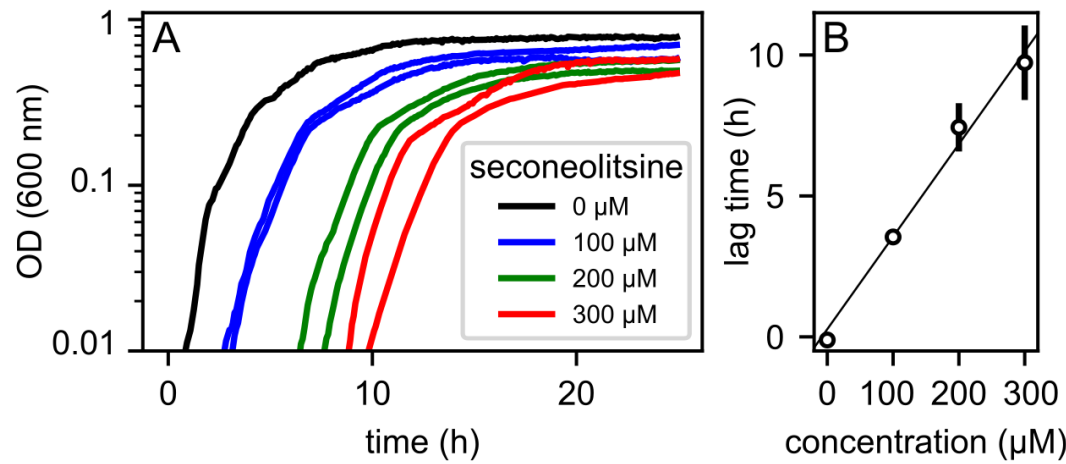

Fig. 1: Antibiotic effect of seconeolitsine on D. dadantii 3937. (A) Growth curves in the presence of increasing amounts of seconeolitsine. (B) Linear increase of the lag time with drug concentration, obtained from a quantitative analysis of growth curves (see Materials and Methods).

We synthesized the TopoI inhibitor seconeolitsine in 13\% yield starting from boldine in a two steps synthesis with neolitsine as intermediate, following the protocol described in the original patent (33) (Supplementary Fig. S1). The resulting powder was solubilized in DMSO, and added to the culture medium of $D$. dadantii 3937 to analyse its inhibitory effect on bacterial growth. We anticipated that the required concentrations might be higher with this Gram-negative bacterium than previously seen with $S$. pneumoniae (31), and this was indeed observed. As with the latter, growth was severely impeded by increasing concentrations of the drug (Fig. 1A), and a quantitative analysis of the growth curves indicated that the lag time was proportional to the applied concentration (Fig. 1B). These data indicate that the minimum inhibitory concentration (MIC), defined as the minimal concentration required to inhibit growth in an overnight culture, has a value between 100 and 200 $\mu \mathrm{M}$, indeed an order of magnitude higher than for $S$. pneumoniae $(16 \mu \mathrm{M})$. Since the antibacterial properties of the product were not our primary interest, we did not characterize them with more precision. We just note that, in order to attain such high concentrations without adding a toxic amount of DMSO ( $<5 \%$ in volume), the solubility of the drug in the stock solution requires some attention. We confirmed that these high concentration requirements were not due to a defect in our product by running a control experiment on the Gram-positive bacterium Bacillus subtilis, which exhibited a much lower inhibitory concentration similar to that of $S$. pneumoniae (around $20 \mu \mathrm{M}$, Supplementary Fig. S2). 


\section{Seconeolitsine shock increases DNA superhelicity in $D$. dadantii cells}

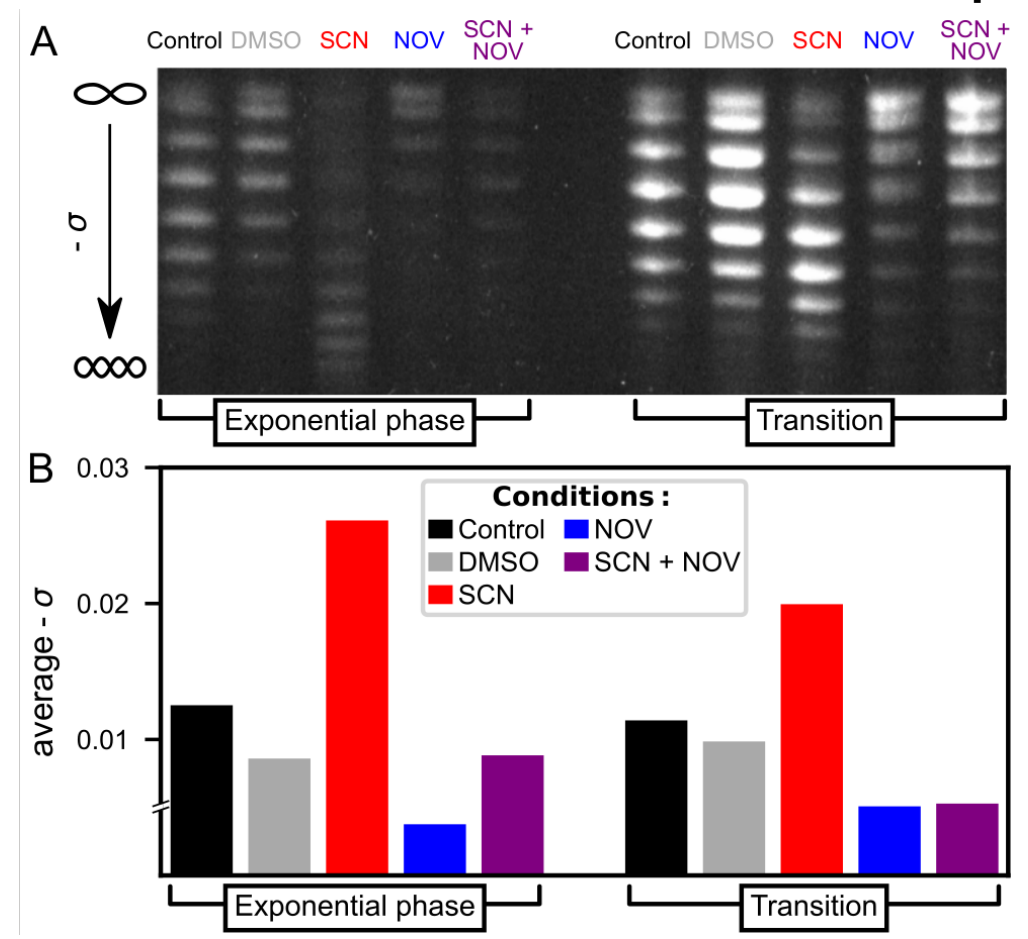

Fig. 2: A seconeolitsine ( $\mathrm{SCN}$ ) shock at $50 \mu \mathrm{M}$ concentration induces an increase of SC in cellular DNA after 15 min. Conversely, a novobiocin (NOV) shock induces DNA relaxation. (A) Agarose-chloroquine gels of pUC18 plasmids isolated from $D$. dadantii 3937 cells. At the employed concentration of chloroquine, the downward migration increases with SC level, and the SC increase induced by seconeolitsine can be fully resolved. (B) Average negative SC level computed from the quantification of the topoisomer distribution. Note that the most relaxed fraction of the topoisomer distribution in presence of novobiocin was not fully resolved, preventing an exact estimation of the relaxation magnitude in these samples, hence the discontinuity indicated in the y-axis.

We then wished to apply a seconeolitsine shock at a lower dosage on D. dadantii 3937 cells, in order to induce a rapid SC increase while affecting the overall cellular physiology as weakly as possible. Using a concentration of $50 \mu \mathrm{M}$ (with a ratio to the MIC comparable to that used in $S$. pneumoniae) indeed did not produce any observable effect on the growth of exponentially growing cells (Supplementary Fig. S3), but lead to a significant shift in the distribution of topoisomers of the pUC18 plasmid extracted 15 min after the shock (Fig. 2A). This time delay was previously chosen to monitor the impact of novobiocin in $D$. dadantii $(16,32,38)$. The quantification of the observed profiles indicates an average increase of negative SC, $\Delta \sigma=-0.014$ in exponential phase and $\Delta \sigma=-0.009$ at the transition to stationary phase, as we expected after an inhibition of the relaxing activity of TopoI by the drug (quantified topoisomer distributions are available in Supplementary Fig. S4). The weaker effect observed at the latter stage was also expected since both gyrase and TopoI are more active in the exponential phase $(8,10,39)$. In both phases, the sharp increase in SC induced by seconeolitsine is in clear opposition to the relaxation induced by novobiocin.

One putative caveat previously identified in absence of TopoI activity (in top $A$ mutants) is the formation of topological domains with a local accumulation of negative SC in plasmids; as a result, the SC level measured on the latter may deviate from that of the chromosome (27-29). We ruled out this possibility in the data of Fig. 2 with three observations: (i) that reported behaviour appeared only when the rotation of the plasmid was hampered by the presence of a gene anchored to the cell membrane by the transcription-translation machinery (40), which is not the case of pUC18; (ii) it 
led to the appearance of a bimodal distribution of topoisomers with a subpopulation of highly negative SC (29), which is not observed here; (iii) we included additional samples treated with both antibiotics, which also present a unimodal distribution shifted to an intermediate SC level between those obtained with each drug (Fig. 2 A and B, rightwards lanes), consistent with a simultaneous steady-state activity of both enzymes on the plasmid rather than a transcription-coupled local accumulation of negative SC (29). Altogether, under all expectations, the observed distributions reliably reflect an increase of the cellular SC level, due to the reduced global activity of TopoI on DNA, equally affecting the chromosome and the plasmids.

\section{Transcriptional response of selected promoters}

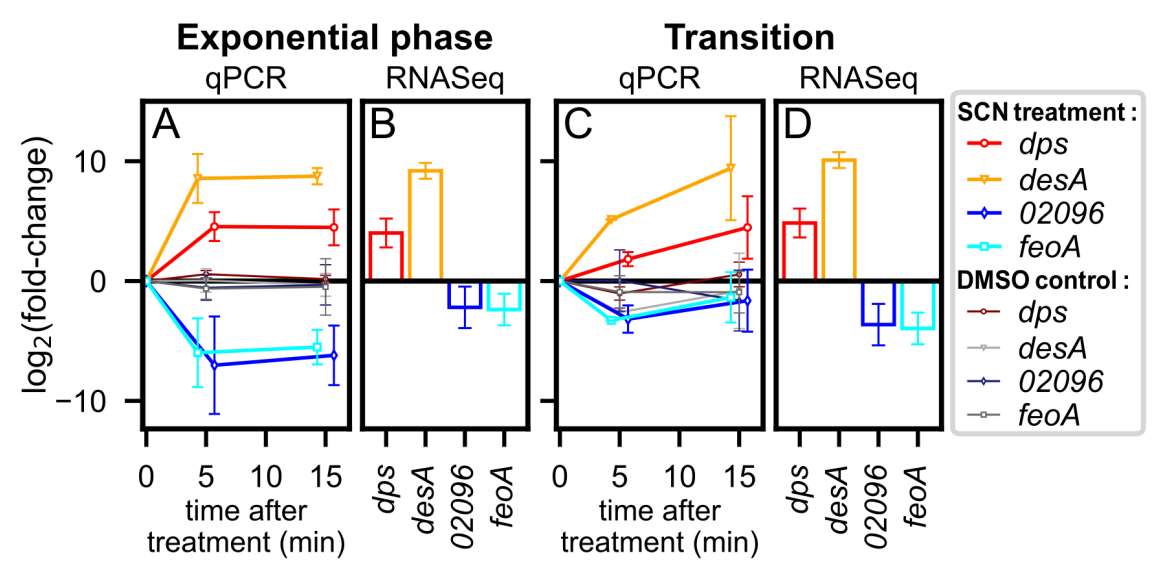

Fig. 3: Kinetics of promoter activation (dps, desA) or repression (Dda3937_02096, feoA) by seconeolitsine (SCN) shock. Gene expression levels were measured in exponential phase (A and B) and at the transition to stationary phase (in C and D), either by qRT-PCR (5 and 15 minutes post-shock, coloured markers and thick lines in A and C) or by RNA-Seq (after 15 min incubation with seconeolitsine, B and D). Control datapoints obtained after incubation with the same volume of pure DMSO solvent are indicated as thin lines, and exhibit no detectable effect. All error bars shown indicate 95\% confidence intervals, obtained with 3 biological replicates (qRT-PCR) or from RNA-Seq analysis.

Based on these observations, we analysed the transcriptional effect of the seconeolitsine shock using RNA-Seq and qRT-PCR validation of selected genes. For simplicity, we first illustrate the kinetics of transcriptional response of four genes that strongly respond to seconeolitisne: the $d p s$ gene encoding the NAP Dps, which is possibly the most abundant DNA-binding protein in stationary phase (41) and condenses the chromosome under conditions of resource scarcity or stress; the $\operatorname{des} A$ gene involved in efflux systems; a gene of unknown function (accession number Dda3937_02096); and feoA involved in iron transport. In exponential phase (Fig. 3A), these genes react very quickly $(5 \mathrm{~min})$ and in opposite manners. The response measured by RNA-Seq after 15 min (B) was entirely consistent with those of qRT-PCR (A); in the latter, we confirmed that DMSO used as solvent, triggers no detectable transcriptional response (thin lines), indicating that seconeolitsine is indeed the active molecule. The same effects were observed at the transition to stationary phase (Fig. $3 \mathrm{C}$ and D), indicating a growth phase-independent regulation. The functions of these strongly responsive genes suggest that they are part of a mechanism of drug-response by the bacteria, which is not surprising given the antibacterial nature of the drug. On the other hand, SC modulates gene expression in a global but usually milder manner (42), as can be observed in Fig. 4 with genes expected to respond specifically to SC variations. 


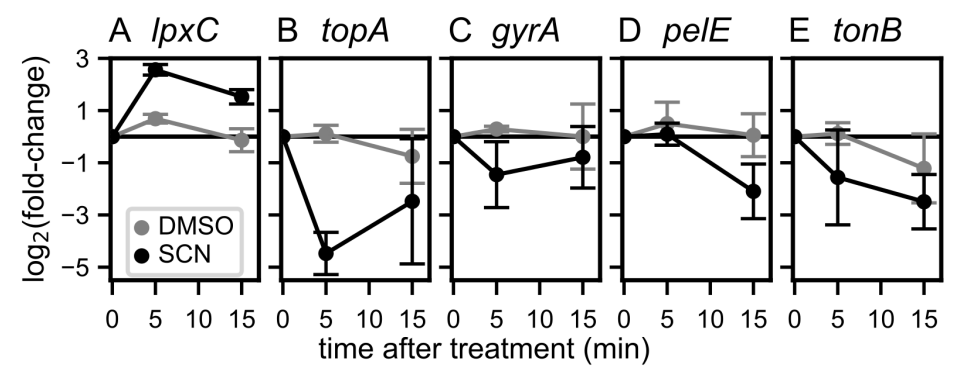

Fig. 4: Effect of seconeolitsine shock on various genes expected to respond to variations of SC: (A) lpxC; (B) topA; (C) gyrA; (D) pelE; (E) tonB. The expression was measured by qPCR 5 and 15 minutes after the shock in exponential phase (black dots). Control datapoints treated with the DMSO solvent are shown (grey dots).

The lpxC gene illustrates some difficulties encountered when analysing SC-controlled regulation. That gene was previously identified as particularly stable in presence of various changes of environmental conditions, and because of this apparent lack of regulation, was considered as a suitable internal normalizer for qRT-PCR experiments with $D$. dadantii (43). However, lpxC was later found to be sensitive to DNA relaxation by novobiocin $(16,38)$. Similarly here, its expression is increased by seconeolitsine treatment in both growth phases, as observed in both qRT-PCR experiments (Fig. 4A) and RNA-Seq data (Tab. S2), the former being either calibrated by concentration gradient or using rроA as internal normalizer. This example shows that SC may affect the expression of a large class of promoters, possibly even those lacking a direct dependence on transcription factors since it modulates the direct interaction of RNAP with promoter DNA (8). The expression of rpoA, on the other hand, appeared stable in the investigated conditions and it was used as normalizer for all qRT-PCR data presented.

We then investigated the response of topoisomerase genes. The topA gene was found to be weakly repressed by the shock (Fig. 4B), in agreement with observations in S. pneumoniae (31). The authors of the latter study analysed the kinetics of SC homeostasis, with a strong initial SC increase followed by a partial relaxation. However, it must be noted that the observed rapid response of the top $A$ gene in both species contradicts the behaviour expected based on that mechanism, since, in analogy to the homeostatic activation of the $g y r A / B$ genes by gyrase inhibition (4), we would have expected an activation in the synthesis of TopoI (24). This repression also contrasts with the activation of top $A$ observed in E. coli after an increase in negative SC induced by oxolinic acid (44), but those experiments were carried in a gyrase mutant strain where the basal SC level is likely more relaxed than in the wild-type strain. In the latter case, the shock might thus restore a SC level close to the physiological one where the expression of top $A$ is maximal (hence the activation), in contrast to our experiment, where the cells start from the physiological level and reach a higher level that may be suboptimal for top $A$ expression (hence the repression). Altogether, SC homeostasis in response to TopoI inhibition is probably not primarily achieved by the regulation of top $A$ expression. Among other topoisomerases, we observed a slight repression of gyrA expression (Fig. $4 \mathrm{C}$, not significant in the less sensitive RNA-Seq data) as well as a possible activation of gyrase inhibitors (the Dda3937_01484 gene, associated to this function by sequence homology, was found significantly activated in the RNA-Seq data, but not confirmed by qRT-PCR). No effect on TopoIV genes $(\operatorname{par} C / E)$ was detected. SC homeostasis might thus be based on a rapid reduction of gyrase activity (counterbalancing the inhibition of TopoI), possibly due to these transcriptional effects, but also likely via post-transcriptional mechanisms (e.g., through the ATP/ADP ratio), as occurs in response to many known stress conditions $(8,12)$. 
We looked at the pelE gene, which encodes a major virulence factor of $D$. dadantii responsible for plant cell wall degrading activity, and is strongly repressed by novobiocin (32). pelE was found to be repressed by seconeolitsine in exponential phase (Fig. 4D), and not significantly affected at the transition where TopoI activity is weaker. The fact that this gene is repressed by both novobiocin (relaxation) and seconeolitsine (SC increase) suggests that the expression is optimal at the natural $\mathrm{SC}$ level, consistent with the tight regulation of this level in the cell (21).

Finally, we investigated the $\operatorname{ton} B$ gene, involved in iron siderophores and vitamin $\mathrm{B}_{12}$ transport at the cell membrane. This gene was previously found to be repressed by an increase of SC induced by anaerobiose in both $E$. coli and $S$. enterica, and this repression was relieved by a novobiocin treatment restoring a SC level close to the physiological one (45). Similarly here, we observed a strong repression of $\operatorname{ton} B$ expression by seconeolitsin in both qRT-PCR experiments (Fig. 4E) and RNA-Seq data (Tab. S2) in exponential phase, giving further support to the repressive effect of strongly negative SC levels on that promoter.

\section{Global transcriptional effect of seconeolitsine shock}

A comparison in the response of several genes confirms that qRT-PCR and RNA-Seq results are well correlated (Supplementary Fig. S5), leading us to analyse the transcriptomic results at a larger scale. Such an analysis is particularly useful for SC-related regulation, which affects many genes in a quantitatively mild manner, because it can unravel global regulatory patterns undetectable at the level of individual promoters $(16,31)$. While most previous studies of gyrase inhibition in Gram-negative bacteria were carried in exponential phase only $(14-16,19)$, we have measured the response to seconeolitsine treatment in the two stages of growth, in each case 15 min after the shock. The lists of differentially expressed genes in either growth phase are given in Supplementary Tab. S2).
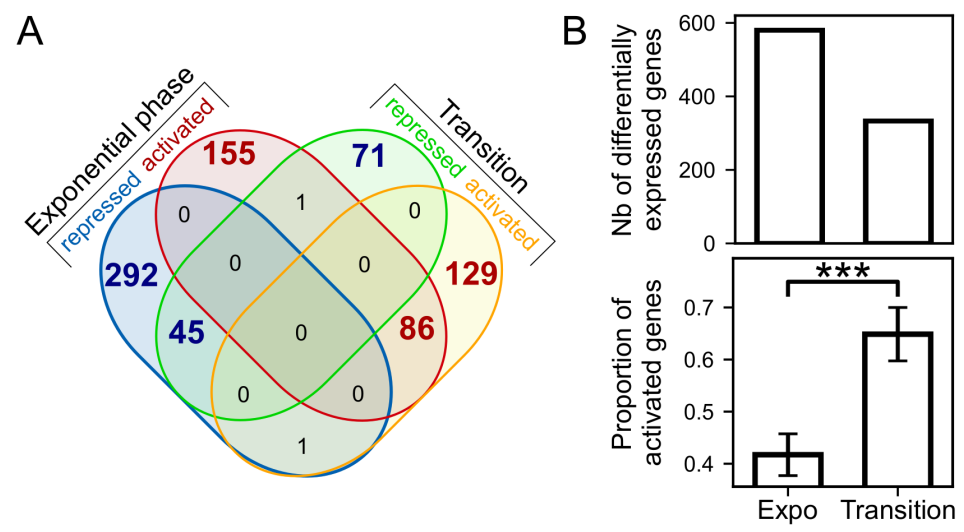

Fig. 5: Global response of the D. dadantii genomic expression to a seconeolitsine shock. (A) Venn diagram of significantly activated or repressed genes at the two stages of growth. (B) Total number of differentially expressed genes (among 4260 genes in total, top) and proportion of activated genes among them (bottom).

The distributions of affected genes are provided in Fig. 5. The shock has a significant impact on around $13 \%$ of the genome in the exponential phase, and about $7 \%$ at the transition to stationary phase. Remarkably, only a small minority of the genes respond significantly in both phases, in which case the response goes in the same direction, whereas many genes respond significantly only in one phase (e.g., pelE). This behaviour was expected since the chromosome conformation (including SC level) and topoisomerase activities are quite different in exponential phase vs transition $(10,39)$. Among differentially expressed genes, the proportion of activated vs repressed 
ones is considerably higher at the transition than in exponential phase (Fig. 5B). The interpretation of these observations is the object of the Discussion below.
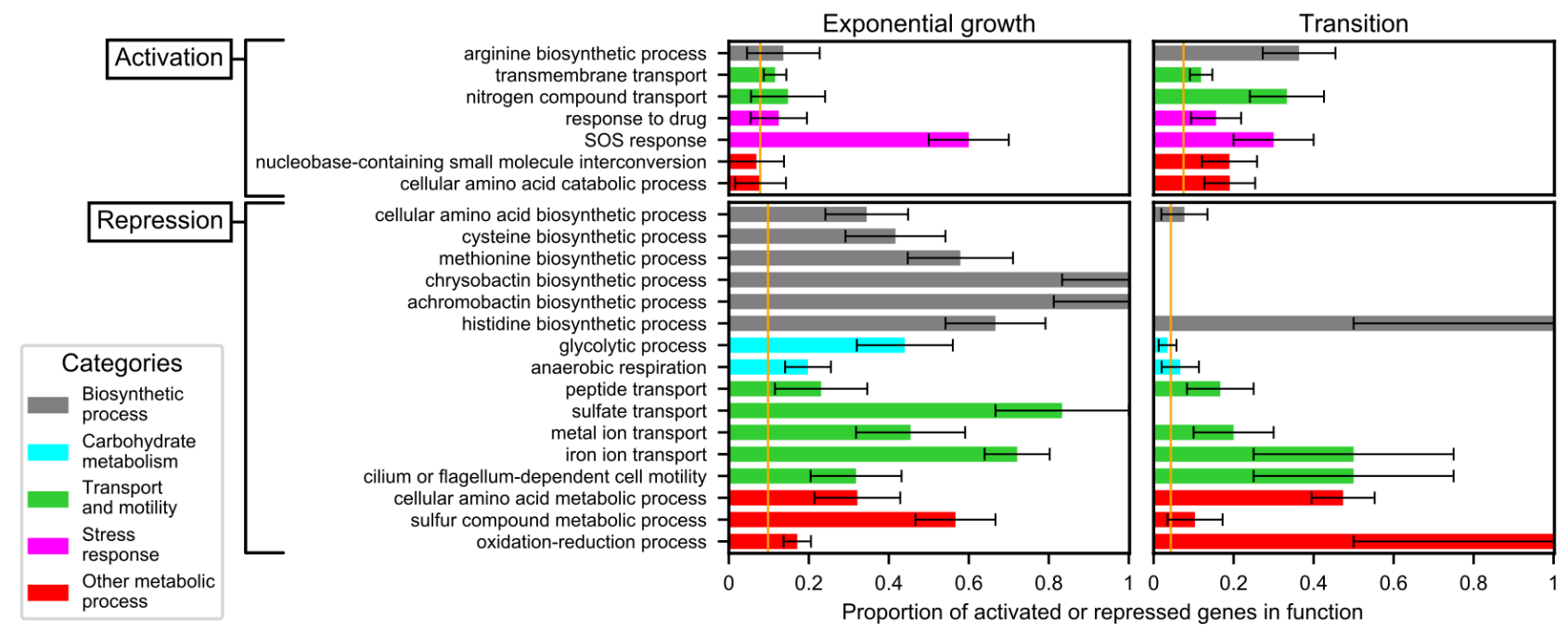

Fig. 6: Functional enrichment analysis of activated (top) or repressed (bottom) genes, during a shock in exponential (left) or transition to stationary phase (right). Each bar indicates the proportion of differentially expressed genes in the considered function (with a $95 \%$ statistical confidence interval), which can be compared to the genomic average (orange vertical lines): the considered function is enriched if the confidence interval does not cross the orange line. Colours indicate the repartition in broad functional groups.

In previous studies, DNA relaxation was shown to regulate the expression of the genome in a functionally scattered way, with limited enrichment in specific regulatory pathways (42). We therefore analysed if the same is true of the seconeolitsine shock (Fig. 6). Indeed, relatively few Gene Ontology (GO) categories exhibit a strong systematic response, and they belong to very diverse functional groups. Expectedly, the most present pathways are related to (i) metabolism and biosynthesis, as already observed during DNA relaxation (14), which are affected differently in the two phases (see grey, blue and red groups in Fig. 6); and (ii) transport and efflux systems, which may, in part, participate in the cellular response to the drug, and are mostly affected similarly in the two phases (green group in Fig. 6). We also noted a strong activation of the iron metabolism pathway. But most importantly, these enriched functions comprise less than $40 \%$ of the total number of differentially expressed genes, showing that most genes are regulated separately rather than within their entire functional category. This feature is characteristic of the "analogue" regulation mode by global changes of the chromosome conformation (42), which affects genes according to their spatial localisation and organisation, in contrast to the classical "digital" regulation by transcriptional regulatory networks, where each regulator often binds many promoters within a functional group. Therefore, we now look in more detail at such spatial organisational features of the global pattern of expression. 


\section{Spatial organisation of promoters sensitive to seconeolitsine shock}

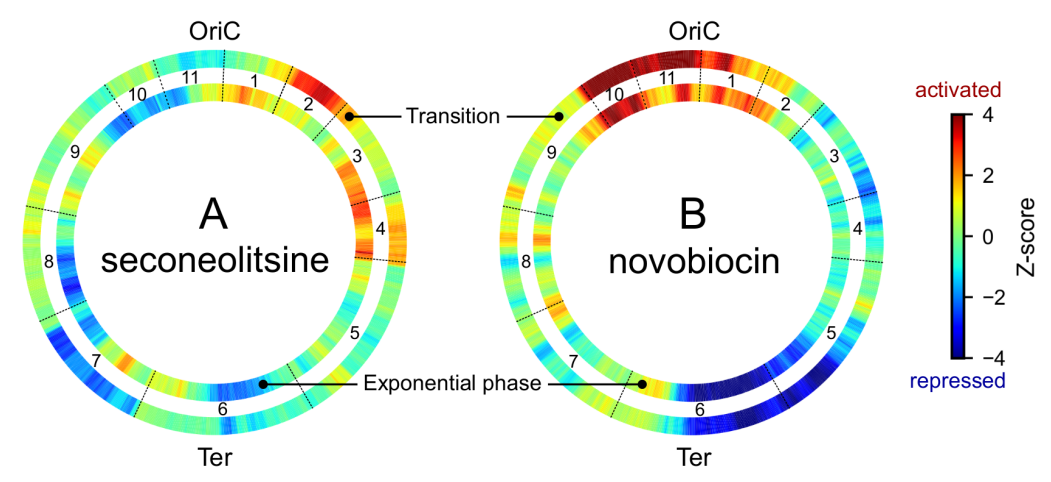

Fig. 7: Distribution of genomic regions enriched in activated (red) or repressed (blue) genes, in exponential phase (internal wheels) or transition to stationary phase (external wheels), during TopoI inhibition by seconeolitsine (A) or gyrase inhibition by novobiocin (B). The colours represent the statistical significance of the proportion of activated over repressed genes in sliding 500-kb windows (Z-score $>2$ or $<-2$, respectively); if the number of differentially expressed genes in the window is low, the Z-score remains close to 0 and appears in green. 11 domains of coherent expression (CODOs) previously identified (16) are indicated.

We started by representing the large-scale distribution of regions enriched in activated or repressed genes along the chromosome (Fig. 7). Strikingly, whereas these regions are almost identical in the two investigated growth phases during a novobiocin shock (right), they are essentially different during a seconeolitsine shock (left), suggesting that, while the large-scale distribution of gyrase activity is similar in the two growth phases, that of TopoI is growth phase-dependent. In line with some observations above, this result clearly shows that the response of promoters to SC variations is not an intrinsic property, but depends on the conditions of the experiment, in this case two different growth phases, characterized in particular by different basal SC levels and spatial distributions of topoisomerase activities.

Previous analyses of $D$. dadantii transcriptomes led to the definition of eleven domains of coherent stress-response, termed CODOs $(16,45)$, which harbour distinct DNA physical properties, are differentially regulated by NAPs and novobiocin, and respond coherently to various stress signals encountered during plant infection. These domains are indicated in Fig. 7 (black boundaries between the wheels), and in many cases, coincide with patterns of TopoI activation/repression. As an example, domain 7 (bottom left) harbouring several virulence genes (type VI secretion systems, flagellar and chemotaxis operons) is repressed by TopoI inhibition at the transition to stationary phase. Interestingly, the same effect is observed when the bacteria are subjected to an osmotic shock at this stage of growth $(16,45)$, which also triggers an increase in negative SC $(16,32)$, and mimics the physiological conditions encountered at the beginning of the maceration phase of plant infection (46). Other domains are repressed in exponential phase (domain 10), or activated either in exponential phase (domain 4) or at the transition (domain 2). Again, this latter observation is consistent with the effect of an osmotic shock, which down-regulates catabolic activity in general and specific stress-responsive genes in domain 2 in particular (16). In summary, although the physical nature and the mechanisms underlying the emergence of these domains remain to be clarified, the transcriptional effect of seconeolitsine gives further support to the notion that they reflect an architectural ordering of the chromosome involving SC and affecting its expression, in line with comparable observations in S. pneumoniae (47). 


\section{Global and local effects of gene orientation}

A notable feature of the large-scale expression pattern (Fig. 7) is that, while the gyrase inhibition pattern is characterized by a clear ori/ter vertical asymmetry (B) possibly reflecting a non-random ordering of genes along the OriC-Ter axis associated to a gradient in gyrase activity (48), the TopoI inhibition pattern rather displays an approximate left/right replichore asymmetry (A). However, a statistical comparison of the proportions of activated genes did not exhibit any global difference between the left and right replichores, suggesting that if the role of TopoI differs between them, this difference is rather localised in specific regions. Rather, we did find a higher proportion of activated genes on the lagging vs leading strand on transition to stationary phase (Supplementary Fig. S6), suggesting that TopoI is more important to dissipate negative supercoils on the leading strand (considering both replichores, i.e., with RNAP and DNA polymerase translocating either in the same or in opposite directions). A putative explanation is that the topological constraints might be weaker on the lagging strand where replication does not proceed continuously. However, this effect is only observed at the transition to stationary phase and is therefore difficult to interpret, since replication is more active in the exponential phase. A putative explanation is that negative supercoils from the replication machinery acting on RNAP might be predominantly handled by TopoIV rather than TopoI in the latter.

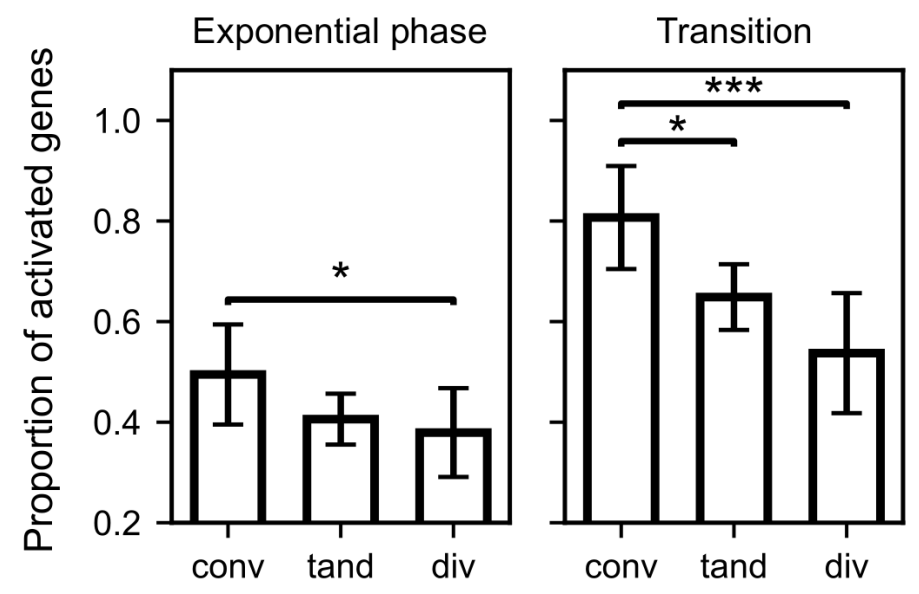

Fig. 8: Gene orientation-dependent transcriptional response to seconeolitsine. The proportion of activated genes (among differentially expressed ones) is significantly higher among those located between convergent (conv) than divergent (div) neighbours, both in exponential phase (left) and at the transition to stationary phase (right). Tandem (tand) genes exhibit intermediate values. Number of differentially expressed genes (conv, tand, div): 57, 205 and 67 in exponential phase, and 97, 362 and 116 at the transition, respectively.

We then focused on a more local scale, and investigated a possible relation between neighbouring gene orientations and the effect of seconeolitsine. Such a relationship was expected as TopoI binds the regions located between divergent operons more actively than others, because these accumulate transcription-generated negative supercoils (49) as occurs at the leu promoter of $S$. enterica where this mechanism was discovered $(29,40)$. The orientation of a gene is here defined by the coding DNA strands of its two neighbours relative to it (in the case of tandem genes, the two neighbours belong to the same strand, which can either be the same as the considered gene or the opposite one). Fig. 8 shows that the expected dependence is indeed observed in both growth phases, with genes located between divergent neighbours being significantly more repressed by TopoI inhibition compared to convergent ones. This observation, made at the scale of the entire genome, shows that the regulatory mechanism uncovered at the leu locus (50) is widespread in the D. dadantii genome 
(and likely in other Gram-negative bacteria). The significant difference between convergent and tandem genes also indicates that TopoI probably is less active in the former regions, likely because of locally generated positive supercoils. The fact that these effects are stronger at the transition to stationary than in exponential phase (where TopoI is more active, see above) suggests that the local accumulation of negative supercoils due to transcription has a stronger regulatory effect when the level of global SC subsides because of reduced topoisomerase activity, as discussed below.

\section{Discussion}

\section{Supercoiling-sensitivity is not an intrinsic property of promoters}

A. Exponential phase

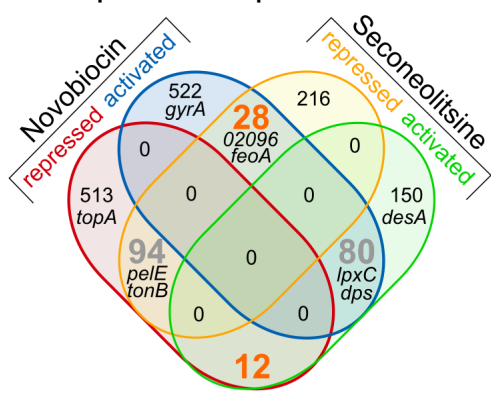

B. Transition

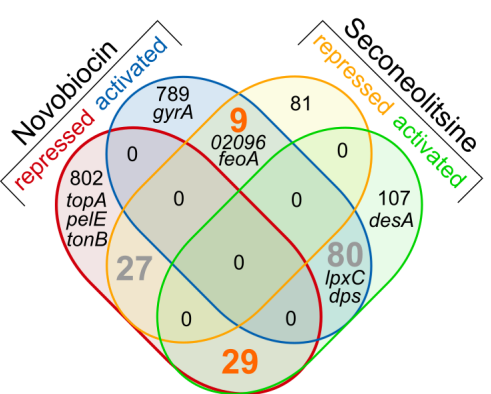

Fig. 9: Venn diagrams of genomic response to novobiocin and seconeolitsine, in either exponential phase (A) or transition to stationary phase (B). Selected genes are indicated in their respective categories. 02096 is the gene with accession number Dda3937_02096.

We have collected the first transcriptomic response to an increase of SC due to TopoI inhibition in a Gram-negative bacterium. Since all previous analyses in these species involved the opposite variation, DNA relaxation induced by gyrase inhibition, we wished to compare these complementary responses, in order to refine our understanding of the notion often referred to as the "supercoiling-sensitivity" of promoters.

Fig. 9 shows that, among genes responding to one of the drugs, the large majority does not respond to the other: genes appearing as sensitive to DNA relaxation are therefore essentially different from those sensitive to an increase of SC. This observation is possibly affected by the limited sensitivity of the RNA-Seq experiment, where some genes confirmed by qRT-PCR (pelE, gyrA) fell below the threshold of statistical significance. Among the genes responding to both drugs, most of them do in the same direction, including some belonging to stress-response functions of the cells possibly via SC-independent regulatory pathways (such as $d p s$ or $\operatorname{des} A$ ) but also some indeed regulated by SC (such as pelE, topA or tonB). Finally, a remarkably low number of genes respond in opposite directions to the two drugs, as would yet be naively expected from promoters exhibiting an intrinsic property of "supercoiling-sensitivity". Note that the latter proportions of similar vs opposed responses to the two drugs were quite comparable in $S$. pneumoniae cells in exponential phase (but more genes were sensitive to both, Supplementary Fig. S7).

These observations probably reflect a combination of factors of different levels of complexity involved in SC-induced transcriptional regulation. In the simplest view, a promoter has been termed "supercoiling-sensitive" if its expression changes as a function of the average SC level of the cell, as can be measured from chloroquine-agarose gels of the type used above. This is a strongly simplified model (see the upcoming paragraphs), but it is already sufficient to explain a difference in sensitivity to gyrase and TopoI inhibition, or a similar response to the two drugs, since the range 
of SC levels reached in the two cases are very different, and the promoter response curve might not be monotonous in that entire range, especially since the physiological SC level is under tight selective pressure (21) and may be optimal for the expression of many promoters. This simplified model is developed further in the next section; we now mention two major factors that further enhance the complexity of SC effects on global transcription.

First, equating the SC level experienced by all promoters with a single average value measured from a topoisomer gel is a crude approximation, because it neglects the spatial heterogeneity of the distribution of supercoils along the chromosome, due to the division of the latter into topological domains (51). This heterogeneity is partly due to the selective activity of gyrase (52), which recognises high-affinity sites along the chromosome in addition to its unspecific binding capacity, whereas TopoI does apparently not target a well-defined sequence motif, in spite of a preference for single-stranded DNA and thus presumably to AT-rich regions $(1,53)$. This difference in sequence selectivity might, in part, explain the higher stability of the expression pattern following novobiocin inhibition compared to TopoI inhibition (Fig. 7). A second factor is the heterogeneous density of supercoils locally generated by transcription, especially in regions located between convergent and divergent operons, which exhibit a preference for gyrase and TopoI respectively, as measured by ChIP-Seq $(38,49,52)$, giving rise to the response observed on Fig. 8. The latter observation relies on genomic signatures (gene orientations) which provide a convenient yet qualitative proxy for the distribution of local SC levels, which is currently not directly accessible with sufficient resolution (54). Based on these spatial effects and probably others (in particular related to replication), the picture of a global chromosomal SC increase or relaxation due to the inhibition of either topoisomerase enzyme appears as somewhat misleading for the understanding of transcriptional regulation by SC: the response of a promoter to SC variations may be expected to depend not only on its own sequence, as an analogy to transcription factors sequence motifs would naively suggest, but also on its genomic context.

A second complexity factor is the dynamical repartition of total SC into contributions that affect transcription in different manners, in particular into constrained and unconstrained SC fractions, and into twist and writhe. These contributions cannot be easily distinguished by conventional chloroquine-agarose gels, and are yet affected very differently by the two main considered topoisomerase enzymes. DNA gyrase introduces supercoils by crossing two distal loci coming into close spatial proximity, i.e., predominantly introduces writhe (55). In contrast, TopoI cleaves only a single strand of negatively supercoiled DNA experiencing transient denaturation bubbles, i.e., predominantly removes an excess of negative twist (1). Although these two contributions are in constant equilibrium and affect each other, the relationship of the two enzymes with RNAP activity thus presumably differs owing to subtle dynamical effects. In turn, these processes may be affected by many factors, both global, such as growth medium and physico-chemical conditions, and local, such as the binding kinetics of NAPs and other proteins at gene promoters, which are usually unknown in the cellular context. As a result, it seems very difficult to integrate this level of mechanistic detail into a predictive regulatory model applicable to in vivo genomic data. 


\section{A qualitative model for the response of bacterial promoters to variations of DNA supercoiling}
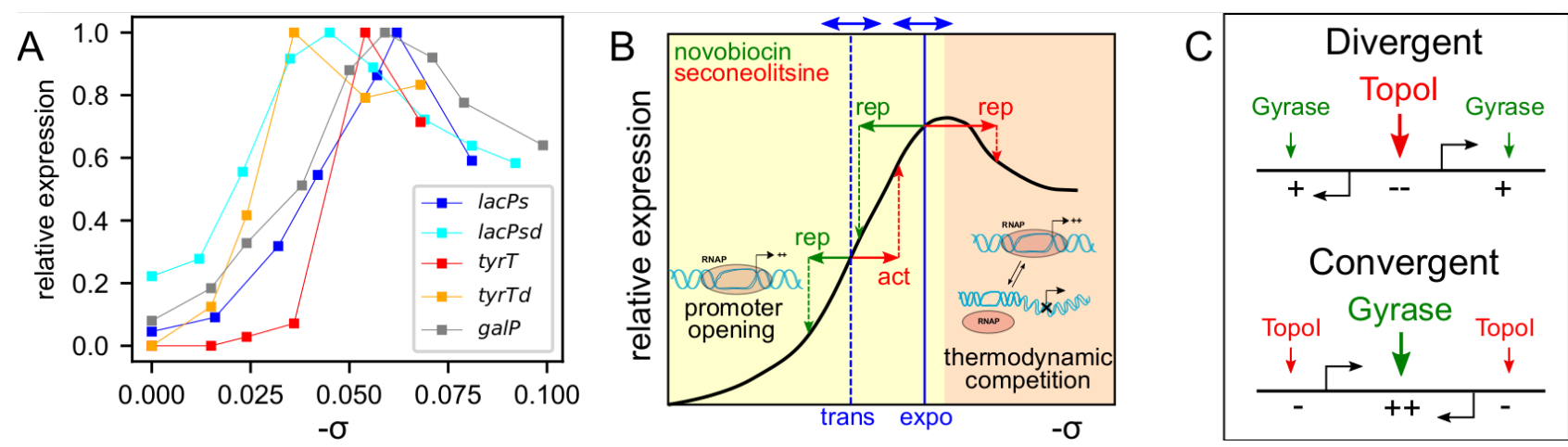

Fig. 10: (A) Regulation of various bacterial promoters by SC in vitro. The employed native promoters encode either stable (tyrT) or messenger RNAs ( galP), whereas lacPs is a mutant promoter derived from lacP. tyrTd and lacPsd are mutant versions of tyrT and lacPs, respectively $(7,11,56)$. (B) Qualitative regulatory model summarizing the data of A (black solid line) with putative mechanisms: promoter DNA opening for open-complex formation (yellow background) (17) and thermodynamic opening competition (orange background, see text) (57). Physiological SC levels valid for many bacteria in exponential or transition to stationary phase are indicated in blue, with double arrows symbolizing limited precision and species-dependent variability (58). The expected regulatory effect of an antibiotic shock in either phase is indicated in green. (C) Model of orientation-dependent binding of topoisomerases, and subsequent transcriptional regulation by TopoI inhibition (adapted from (29)).

In spite of aforementioned limitations of our knowledge, is it possible to propose at least a qualitative model of transcriptional regulation, depending only on the experimentally accessible average SC level measured in a given culture condition, and recapitulating the most notable features observed in transcriptomes obtained with both gyrase and TopoI inhibitors?

In order to eliminate uncontrolled parameters and focus on the most generic features of SC-dependent transcriptional regulation, it is useful to analyse in vitro transcription data, where genes are expressed on plasmids with minimal influence of genomic context or regulatory proteins, and where SC is modified in a controlled way. Fig. 10A recapitulates several available datasets of this kind $(7,11,56)$ obtained with a broad sampling of SC levels comprising typical physiological levels, either in standard conditions (from -0.04 to -0.06), upon gyrase inhibition (lower negative SC levels) and upon TopoI inhibition (higher negative SC levels). The employed promoters belong to different promoter families, either from stable RNAs (tyrT) and mutant promoters derived thereof $($ tyr $T d)$, or promoters of protein-encoding genes $(\mathrm{galP})$ or derivatives of lacP (lacPs, lacPsd). And yet apart from conspicuous differences between these curves, a similar pattern is clearly and repeatedly observable: the expression is very low on an entirely relaxed DNA template, then increases drastically and monotonously until reaching maximal expression at a (promoter-dependent) optimal SC level close to the physiological level in exponential phase $(\approx$ -0.06), then decreases at higher SC levels. This behaviour is schematized in Fig. 10B, where the horizontal axis is voluntarily left without quantitative values. The two background colours highlight the two regulation regimes with putative associated mechanisms: the initial activation curve is likely due to the SC-induced reduction of DNA opening free energy during open-complex formation, which occurs preferentially at the highly AT-rich region starting at the -10 promoter element where the transcription bubble is formed (17); the decrease is more complex and either due to the opening of secondary sites competing with the -10 element (57), or to a reduction in processive initiation due to an excessive stability of the open-complex resulting in more abortive 
transcripts (6). Accordingly, while a modification of the AT-richness of the promoter sequence downstream of the -10 element (in lacPsd and tyrTd compared to lacPs and tyrT, respectively) clearly shifts the activation curve horizontally in a predictable manner (17), the second part of the curve is more variable, and the position of the maximum differs significantly from one promoter to the other (7). Based on this empirical model, the regulatory effect of a SC variation (e.g., due to an antibiotic) is then expected to depend both on the initial global SC level in the cell, and on the direction and magnitude of the SC change. Approximate values of the average SC level in exponential or transition to stationary phase are indicated in blue, with exact values varying between species (58).

During a relaxation shock, in either phase, the expression rate is predicted to be shifted leftwards to a lower level. The effect of the shock is thus relatively similar in both phases, explaining the comparable pattern of expression observed with novobiocin (Fig. 7 right). Note that while all promoters become more difficult to open after DNA relaxation, the effective total expression level in the cell does not necessarily decrease, since it is rather set by the availability of RNAP for which promoters are in competition (59), and these might consequently still be either activated or repressed according to their individual curves (as in Fig. 10A).

During a seconeolitsine shock, the situation is different. At the moderate SC level experienced during transition to stationary phase, the SC increase (rightwards shift) induced by TopoI inhibition is expected to induce the expression rate of most promoters; in contrast, in exponential phase, the SC level is already close to the maximum of the curve, and the shock thus tends to reduce the expression level of many individual promoters, although this response is likely more sensitive to the exact optimal level of each promoter and thus more complex.

Although very simplified, this analysis from in vitro data might thus contribute to several notable observations that we have made from our data: (i) many promoters, such as pelE, $\operatorname{lp} x C$, tonB and $d p s$, respond to novobiocin and seconeolitsine in the same direction, suggesting a non-monotonous SC-activation curve (Fig. 9); (ii) the expression pattern associated to seconeolitsine is more condition-dependent than that of novobiocin (Fig. 7); (iii) seconeolitsine mostly represses promoters in exponential phase, and activates them at the transition to stationary phase (Fig. 5B).

To avoid any over-interpretation of this qualitative model, we have included a model diagram describing the role of relative gene orientations in the transcriptional effect of TopoI inhibition (Fig. 10C), directly inspired by previous models $(29,60)$, and illustrating the role of spatial SC heterogeneities along the chromosome mentioned in the previous paragraph, in addition to the average level illustrated in Fig. 10B. Altogether, this analysis highlights the complexity of this regulation mode, and in particular, suggests that the notion of "supercoiling-sensitivity" of a promoter should be taken with caution. Although some specific promoters like $g y r A / B$ can exhibit a robust invariant response apparently inscribed in their sequence organisation (61), the response of many other promoters to variations of SC might vary (i) in vitro vs in vivo; (ii) depending on the genomic context; (iii) depending on the conditions of the experiment (ionic composition, basal SC level, metabolic state etc.). Thus, in our view, the "supercoiling-sensitivity" should not be considered exclusively as a promoter-specific property, but rather as a potentially widespread behaviour that emerges in a condition-dependent manner. A better understanding of this regulation will require, in particular, a detailed mapping of the distribution of supercoils along the chromosome (54). 


\section{Acknowledgments}

We thank Georgi Muskhelishvili for his suggestions and critical reading of the manuscript, and Florelle Deboudard for technical help.

\section{Funding}

This work was supported by a BQR 2016 grant from INSA Lyon and an Agence Nationale de la Recherche grant (ANR-18-CE45-0006-01).

\section{References}

1. Maxwell,A. and Gellert,M. (1986) Mechanistic Aspects of DNA Topoisomerases. In Anfinsen,C.B., Edsall,J.T., Richards,F.M. (eds), Advances in Protein Chemistry. Academic Press, Vol. 38, pp. 69-107.

2. Zechiedrich,E.L., Khodursky,A.B., Bachellier,S., Schneider,R., Chen,D., Lilley,D.M. and Cozzarelli,N.R. (2000) Roles of topoisomerases in maintaining steady-state DNA supercoiling in Escherichia coli. J. Biol. Chem., 275, 8103-8113.

3. Chen,S.H., Chan,N.-L. and Hsieh,T. (2013) New mechanistic and functional insights into DNA topoisomerases. Annu. Rev. Biochem., 82, 139-170.

4. Menzel,R. and Gellert,M. (1983) Regulation of the genes for E. coli DNA gyrase: homeostatic control of DNA supercoiling. Cell, 34, 105-113.

5. Ohlsen,K.L. and Gralla,J.D. (1992) Interrelated effects of DNA supercoiling, ppGpp, and low salt on melting within the Escherichia coli ribosomal RNA rrnB P1 promoter. Mol. Microbiol., 6, 2243-2251.

6. Wood,D.C. and Lebowitz,J. (1984) Effect of supercoiling on the abortive initiation kinetics of the RNA-I promoter of ColE1 plasmid DNA. J. Biol. Chem., 259, 11184-11187.

7. Lim,H.M., Lewis,D.E.A., Lee,H.J., Liu,M. and Adhya,S. (2003) Effect of varying the supercoiling of DNA on transcription and its regulation. Biochemistry, 42, 10718-10725.

8. Martis B,S., Forquet,R., Reverchon,S., Nasser,W. and Meyer,S. (2019) DNA Supercoiling: an Ancestral Regulator of Gene Expression in Pathogenic Bacteria? Comput. Struct. Biotechnol. J., 17, 1047-1055.

9. Pruss,G.J. and Drlica,K. (1989) DNA supercoiling and prokaryotic transcription. Cell, 56, 521-523.

10. Travers,A. and Muskhelishvili,G. (2005) DNA supercoiling - a global transcriptional regulator for enterobacterial growth? Nat. Rev. Microbiol., 3, 157-169.

11. Auner,H., Buckle,M., Deufel,A., Kutateladze,T., Lazarus,L., Mavathur,R., Muskhelishvili,G., Pemberton,I., Schneider,R. and Travers,A. (2003) Mechanism of transcriptional activation by FIS: role of core promoter structure and DNA topology. J. Mol. Biol., 331, 331-344.

12. Hsieh,L.S., Rouviere-Yaniv,J. and Drlica,K. (1991) Bacterial DNA supercoiling and [ATP]/[ADP] ratio: changes associated with salt shock. J. Bacteriol., 173, 3914-3917.

13. Borowiec,J.A. and Gralla,J.D. (1985) Supercoiling response of the lac ps promoter in vitro. $J$. Mol. Biol., 184, 587-598.

14. Blot,N., Mavathur,R., Geertz,M., Travers,A. and Muskhelishvili,G. (2006) Homeostatic regulation of supercoiling sensitivity coordinates transcription of the bacterial genome. EMBO Rep., 7, 710-715.

15. Peter,B.J., Arsuaga,J., Breier,A.M., Khodursky,A.B., Brown,P.O. and Cozzarelli,N.R. (2004) Genomic transcriptional response to loss of chromosomal supercoiling in Escherichia coli. Genome Biol., 5, R87. 
16. Jiang,X., Sobetzko,P., Nasser,W., Reverchon,S. and Muskhelishvili,G. (2015) Chromosomal 'stress-response' domains govern the spatiotemporal expression of the bacterial virulence program. mBio, 6, e00353-00315.

17. Forquet,R., Pineau,M., Nasser,W., Reverchon,S. and Meyer,S. (2020) Bacterial promoter opening underpins ubiquitous transcriptional regulation by DNA supercoiling. bioRxiv, 10.1101/2020.10.01.322149.

18. Jovanovich,S.B. and Lebowitz,J. (1987) Estimation of the effect of coumermycin A1 on Salmonella typhimurium promoters by using random operon fusions. J. Bacteriol., 169, 4431-4435.

19. Gogoleva,N.E., Konnova,T.A., Balkin,A.S., Plotnikov,A.O. and Gogolev,Y.V. (2020) Transcriptomic data of Salmonella enterica subsp. enterica serovar Typhimurium str. 14028S treated with novobiocin. Data Brief, 29, 105297.

20. Drlica,K. (1992) Control of bacterial DNA supercoiling. Mol. Microbiol., 6, 425-433.

21. Pruss,G.J., Manes,S.H. and Drlica,K. (1982) Escherichia coli DNA topoisomerase I mutants: increased supercoiling is corrected by mutations near gyrase genes. Cell, 31, 35-42.

22. Champoux,J.J. (2001) DNA Topoisomerases: Structure, Function, and Mechanism. Annu. Rev. Biochem., 70, 369-413.

23. Franco,R.J. and Drlica,K. (1989) Gyrase inhibitors can increase gyrA expression and DNA supercoiling. J. Bacteriol., 171, 6573-6579.

24. Seddek,A., Annamalai,T. and Tse-Dinh,Y.-C. (2021) Type IA Topoisomerases as Targets for Infectious Disease Treatments. Microorganisms, 9.

25. Cheng,B., Zhu,C.-X., Ji,C., Ahumada,A. and Tse-Dinh,Y.-C. (2003) Direct interaction between Escherichia coli RNA polymerase and the zinc ribbon domains of DNA topoisomerase I. $J$. Biol. Chem., 278, 30705-30710.

26. Wang,J.C. (1971) Interaction between DNA and an Escherichia coli protein omega. J. Mol. Biol., 55, 523-533.

27. Pruss,G.J., Franco,R.J., Chevalier,S.G., Manes,S.H. and Drlica,K. (1986) Effects of DNA gyrase inhibitors in Escherichia coli topoisomerase I mutants. J. Bacteriol., 168, 276-282.

28. Wang,J.C. and Lynch,A.S. (1993) Transcription and DNA supercoiling. Curr. Opin. Genet. Dev., 3, 764-768.

29. Lilley,D.M.J., Chen,D. and Bowater,R.P. (1996) DNA supercoiling and transcription: topological coupling of promoters. Q. Rev. Biophys., 29, 203-225.

30. García,M.T., Blázquez,M.A., Ferrándiz,M.J., Sanz,M.J., Silva-Martín,N., Hermoso,J.A. and de la Campa,A.G. (2011) New alkaloid antibiotics that target the DNA topoisomerase I of Streptococcus pneumoniae. J. Biol. Chem., 286, 6402-6413.

31. Ferrándiz,M.-J., Martín-Galiano,A.J., Arnanz,C., Camacho-Soguero,I., Tirado-Vélez,J.-M. and de la Campa,A.G. (2016) An increase in negative supercoiling in bacteria reveals topology-reacting gene clusters and a homeostatic response mediated by the DNA topoisomerase I gene. Nucleic Acids Res., 44, 7292-7303.

32. Ouafa,Z.-A., Reverchon,S., Lautier,T., Muskhelishvili,G. and Nasser,W. (2012) The nucleoid-associated proteins H-NS and FIS modulate the DNA supercoiling response of the pel genes, the major virulence factors in the plant pathogen bacterium Dickeya dadantii. Nucleic Acids Res., 40, 4306-4319.

33. Campa,A.G.D.L., Ferrer,M.A.B. and Esteban,M.T.G. (2012) Use of seconeolitsin and n-methyl-seconeolitsin a for the manufacture of medicines. ES2363074B1.

34. Zwietering,M.H., Jongenburger,I., Rombouts,F.M. and van 't Riet,K. (1990) Modeling of the bacterial growth curve. Appl. Environ. Microbiol., 56, 1875-1881.

35. Reverchon,S., Meyer,S., Forquet,R., Hommais,F., Muskhelishvili,G. and Nasser,W. (2021) The nucleoid-associated protein IHF acts as a 'transcriptional domainin' protein coordinating the bacterial virulence traits with global transcription. Nucleic Acids Res., 49, 776-790.

36. Maes,M. and Messens,E. (1992) Phenol as grinding material in RNA preparations. Nucleic 
Acids Res., 20, 4374.

37. Ashburner,M., Ball,C.A., Blake,J.A., Botstein,D., Butler,H., Cherry,J.M., Davis,A.P., Dolinski,K., Dwight,S.S., Eppig,J.T., et al. (2000) Gene Ontology: tool for the unification of biology. Nat. Genet., 25, 25-29.

38. El Houdaigui,B., Forquet,R., Hindré,T., Schneider,D., Nasser,W., Reverchon,S. and Meyer,S. (2019) Bacterial genome architecture shapes global transcriptional regulation by DNA supercoiling. Nucleic Acids Res., 47, 5648-5657.

39. Balke,V.L. and Gralla,J.D. (1987) Changes in the linking number of supercoiled DNA accompany growth transitions in Escherichia coli. J. Bacteriol., 169, 4499-4506.

40. Lynch,A.S. and Wang,J.C. (1993) Anchoring of DNA to the bacterial cytoplasmic membrane through cotranscriptional synthesis of polypeptides encoding membrane proteins or proteins for export: a mechanism of plasmid hypernegative supercoiling in mutants deficient in DNA topoisomerase I. J. Bacteriol., 175, 1645-1655.

41. Verma,S.C., Qian,Z. and Adhya,S.L. (2019) Architecture of the Escherichia coli nucleoid. PLoS Genet., 15, e1008456.

42. Travers,A. and Muskhelishvili,G. (2020) Chromosomal Organization and Regulation of Genetic Function in Escherichia coli Integrates the DNA Analog and Digital Information. EcoSal Plus, 9.

43. Hommais,F., Zghidi-Abouzid,O., Oger-Desfeux,C., Pineau-Chapelle,E., Van Gijsegem,F., Nasser,W. and Reverchon,S. (2011) lpxC and yafS are the most suitable internal controls to normalize real time RT-qPCR expression in the phytopathogenic bacteria Dickeya dadantii. PloS One, 6, e20269.

44. Tse-Dinh,Y.C. and Beran,R.K. (1988) Multiple promoters for transcription of the Escherichia coli DNA topoisomerase I gene and their regulation by DNA supercoiling. J. Mol. Biol., 202, 735-742.

45. Muskhelishvili,G., Forquet,R., Reverchon,S., Meyer,S. and Nasser,W. (2019) Coherent Domains of Transcription Coordinate Gene Expression During Bacterial Growth and Adaptation. Microorganisms, 7.

46. Leonard,S., Hommais,F., Nasser,W. and Reverchon,S. (2017) Plant-phytopathogen interactions: bacterial responses to environmental and plant stimuli. Environ. Microbiol., 19, 1689-1716.

47. de la Campa,A.G., Ferrándiz,M.J., Martín-Galiano,A.J., García,M.T. and Tirado-Vélez,J.M. (2017) The Transcriptome of Streptococcus pneumoniae Induced by Local and Global Changes in Supercoiling. Front. Microbiol., 8, 1447.

48. Sobetzko,P., Travers,A. and Muskhelishvili,G. (2012) Gene order and chromosome dynamics coordinate spatiotemporal gene expression during the bacterial growth cycle. Proc. Natl. Acad. Sci. U. S. A., 109, E42-50.

49. Ahmed,W., Sala,C., Hegde,S.R., Jha,R.K., Cole,S.T. and Nagaraja,V. (2017) Transcription facilitated genome-wide recruitment of topoisomerase I and DNA gyrase. PLoS Genet., 13, e1006754.

50. Chen,D., Bowater,R., Dorman,C.J. and Lilley,D.M. (1992) Activity of a plasmid-borne leu-500 promoter depends on the transcription and translation of an adjacent gene. Proc. Natl. Acad. Sci. U. S. A., 89, 8784-8788.

51. Postow,L., Hardy,C.D., Arsuaga,J. and Cozzarelli,N.R. (2004) Topological domain structure of the Escherichia coli chromosome. Genes Dev., 18, 1766-1779.

52. Sutormin,D., Rubanova,N., Logacheva,M., Ghilarov,D. and Severinov,K. (2019)

Single-nucleotide-resolution mapping of DNA gyrase cleavage sites across the Escherichia coli genome. Nucleic Acids Res., 47, 1373-1388.

53. Tan,K., Zhou,Q., Cheng,B., Zhang,Z., Joachimiak,A. and Tse-Dinh,Y.-C. (2015) Structural basis for suppression of hypernegative DNA supercoiling by E. coli topoisomerase I. Nucleic Acids Res., 43, 11031-11046.

54. Lal,A., Dhar,A., Trostel,A., Kouzine,F., Seshasayee,A.S.N. and Adhya,S. (2016) Genome scale 
patterns of supercoiling in a bacterial chromosome. Nat. Commun., 7, 11055.

55. Bush,N.G., Evans-Roberts,K. and Maxwell,A. (2015) DNA Topoisomerases. EcoSal Plus, 6.

56. Borowiec,J.A. and Gralla,J.D. (1987) All three elements of the lac ps promoter mediate its transcriptional response to DNA supercoiling. J. Mol. Biol., 195, 89-97.

57. Hatfield,G.W. and Benham,C.J. (2002) DNA topology-mediated control of global gene expression in Escherichia coli. Annu. Rev. Genet., 36, 175-203.

58. Rovinskiy,N.S., Agbleke,A.A., Chesnokova,O.N. and Higgins,N.P. (2019) Supercoil Levels in E. coli and Salmonella Chromosomes Are Regulated by the C-Terminal $35^{-} 38$ Amino Acids of GyrA. Microorganisms, 7.

59. Klumpp,S. and Hwa,T. (2008) Growth-rate-dependent partitioning of RNA polymerases in bacteria. Proc. Natl. Acad. Sci., 105, 20245-20250.

60. Liu,L.F. and Wang,J.C. (1987) Supercoiling of the DNA template during transcription. Proc. Natl. Acad. Sci., 84, 7024-7027.

61. Menzel,R. and Gellert,M. (1987) Modulation of transcription by DNA supercoiling: a deletion analysis of the Escherichia coli gyrA and gyrB promoters. Proc. Natl. Acad. Sci. U. S. A., 84, 4185-4189. 


\section{Supplementary Information}

Fig. S1: Synthesis of seconeolitsine from boldine in a two steps reaction with neolitsine as intermediate.

Fig. S2: Growth curves of the Gram-positive bacterium B. subtilis in presence of increasing amounts of seconeolitsine, solvated in a constant ( $5 \%$ vol) volume of DMSO.

Fig. S3: Growth curves of D. dadantii treated with a seconeolitsine shock at $50 \mu \mathrm{M}$ during exponential growth (vertical line), exhibiting no significant effect (multiple black and grey lines indicate replicates of treated and untreated samples, respectively, and are almost indistinguishable). The chosen concentration thus minimizes the metabolic effect of the shock, while inducing a significant increase in SC (Fig. 2). The experiment was carried in M63+glucose (A) and LB (B) culture media.

Fig. S4: Quantification of topoisomer distribution of pUC18 plasmids isolated from D. dadantii. Cells were treated with seconeolitsine $(50 \mu \mathrm{M})$, novobiocin $\left(100 \mu \mathrm{g} \cdot \mathrm{ml}^{-1}\right)$ or both, in exponential (left) and transition to stationary phase (right). The most relaxed fractions were not fully resolved on the chloroquine gel, hence the discontinuity indicated in the $\mathrm{x}$-axis.

Fig. S5: Comparison of gene responses obtained with qRT-PCR and RNA-Seq to seconeolitsine treatment in exponential (left) and transition to stationary phase (right). Pearson's correlation coefficients reached 0.904 with p-value of $6.23 \mathrm{e}^{-7}$ (exponential phase) and 0.917 with p-value of $2.32 \mathrm{e}^{-7}$ (transition). 02096 is the gene with accession number Dda3937_02096.

Fig. S6: Proportion of activated genes (among differentially expressed ones) on the lagging vs leading replicative strand in exponential phase (A) and at the transition to stationary phase (B).

Fig. S7: Venn diagram of the response of $S$. pneumoniae genes to novobiocin and seconeolitsine treatments in exponential phase, computed from the data of (31).

Tab. S1: List of primers used in PCR.

Tab. S2: Response of $D$. dadantii genes to seconeolitsine treatment in exponential and transition to stationary phase. 


\section{List of figures}

Fig. 1: Antibiotic effect of seconeolitsine on D. dadantii 3937. (A) Growth curves in the presence of increasing amounts of seconeolitsine. (B) Linear increase of the lag time with drug concentration, obtained from a quantitative analysis of growth curves (see Materials and Methods).

Fig. 2: A seconeolitsine ( $\mathrm{SCN}$ ) shock at $50 \mu \mathrm{M}$ concentration induces an increase of SC in cellular DNA after $15 \mathrm{~min}$. Conversely, a novobiocin (NOV) shock induces DNA relaxation. (A) Agarose-chloroquine gels of pUC18 plasmids isolated from D. dadantii 3937 cells. At the employed concentration of chloroquine, the downward migration increases with SC level, and the SC increase induced by seconeolitsine can be fully resolved. (B) Average negative SC level computed from the quantification of the topoisomer distribution. Note that the most relaxed fraction of the topoisomer distribution in presence of novobiocin was not fully resolved, preventing an exact estimation of the relaxation magnitude in these samples, hence the discontinuity indicated in the y-axis.

Fig. 3: Kinetics of promoter activation (dps, desA) or repression (Dda3937_02096, feoA) by seconeolitsine $(\mathrm{SCN})$ shock. Gene expression levels were measured in exponential phase (A and $\mathrm{B}$ ) and at the transition to stationary phase (in $\mathrm{C}$ and D), either by qRT-PCR ( 5 and 15 minutes post-shock, coloured markers and thick lines in A and C) or by RNA-Seq (after 15 min incubation with seconeolitsine, B and D). Control datapoints obtained after incubation with the same volume of pure DMSO solvent are indicated as thin lines, and exhibit no detectable effect. All error bars shown indicate $95 \%$ confidence intervals, obtained with 3 biological replicates (qRT-PCR) or from RNA-Seq analysis.

Fig. 4: Effect of seconeolitsine shock on various genes expected to respond to variations of SC: (A) $\operatorname{lpxC}$; (B) topA; (C) gyrA; (D) pelE; (E) tonB. The expression was measured by qPCR 5 and 15 minutes after the shock in exponential phase (black dots). Control datapoints treated with the DMSO solvent are shown (grey dots).

Fig. 5: Global response of the D. dadantii genomic expression to a seconeolitsine shock. (A) Venn diagram of significantly activated or repressed genes at the two stages of growth. (B) Total number of differentially expressed genes (among 4260 genes in total, top) and proportion of activated genes among them (bottom).

Fig. 6: Functional enrichment analysis of activated (top) or repressed (bottom) genes, during a shock in exponential (left) or transition to stationary phase (right). Each bar indicates the proportion of differentially expressed genes in the considered function (with a 95\% statistical confidence interval), which can be compared to the genomic average (orange vertical lines): the considered function is enriched if the confidence interval does not cross the orange line. Colours indicate the repartition in broad functional groups.

Fig. 7: Distribution of genomic regions enriched in activated (red) or repressed (blue) genes, in exponential phase (internal wheels) or transition to stationary phase (external wheels), during TopoI inhibition by seconeolitsine (A) or gyrase inhibition by novobiocin (B). The colours represent the statistical significance of the proportion of activated over repressed genes in sliding 500-kb windows (Z-score $>2$ or $<-2$, respectively); if the number of differentially expressed genes in the 
window is low, the Z-score remains close to 0 and appears in green. 11 domains of coherent expression (CODOs) previously identified (16) are indicated.

Fig. 8: Gene orientation-dependent transcriptional response to seconeolitsine. The proportion of activated genes (among differentially expressed ones) is significantly higher among those located between convergent (conv) than divergent (div) neighbours, both in exponential phase (left) and at the transition to stationary phase (right). Tandem (tand) genes exhibit intermediate values. Number of differentially expressed genes (conv, tand, div): 57, 205 and 67 in exponential phase, and 97, 362 and 116 at the transition, respectively.

Fig. 9: Venn diagrams of genomic response to novobiocin and seconeolitsine, in either exponential phase (A) or transition to stationary phase (B). Selected genes are indicated in their respective categories. 02096 is the gene with accession number Dda3937_02096.

Fig. 10: (A) Regulation of various bacterial promoters by SC in vitro. The employed native promoters encode either stable (tyrT) or messenger RNAs ( galP), whereas lacPs is a mutant promoter derived from lacP. tyrTd and lacPsd are mutant versions of tyrT and lacPs, respectively $(7,11,56)$. (B) Qualitative regulatory model summarizing the data of A (black solid line) with putative mechanisms: promoter DNA opening for open-complex formation (yellow background) (17) and thermodynamic opening competition (orange background, see text) (57). Physiological SC levels valid for many bacteria in exponential or transition to stationary phase are indicated in blue, with double arrows symbolizing limited precision and species-dependent variability (58). The expected regulatory effect of an antibiotic shock in either phase is indicated in green. (C) Model of orientation-dependent binding of topoisomerases, and subsequent transcriptional regulation by TopoI inhibition (adapted from (29)). 